\title{
Spectroscopic Masses for post-AGB Stars and Planetary Nebula Nuclei
}

\author{
Mirosław Schmidt \& Romuald Tylenda \\ Department for Astrophysics, N. Copernicus Astronomical Centre, \\ Rabiańska 8, 87-100 Toruń, Poland
}

\begin{abstract}
We compare the observed positions of post-AGB stars and planetary nebula nuclei in the $\log g-\log T_{\text {eff }}$ diagram. We show that these positions and the resulting stellar masses are not fully consistent with theoretical expectations.
\end{abstract}

Post-AGB stars (hereinafter pAGB) are in a short lived evolutionary transition between the asymptotic giant branch and the planetary nebula phase. The masses of these objects can be estimated from their values of $\log g$ and $T_{\text {eff }}$ determined from spectroscopic studies and compared to theoretical evolutionary tracks. The method is essentially the same as that used for planetary nebula nuclei (hereinafter PNN) (e.g. Méndez et al. 1992, Napiwotzki 1999).

The spectroscopic parameters for 80 pAGB have been taken from an updated database of Schmidt (2001). The database results from a compilation of values published in the literature. The observed parameters are compared to evolutionary tracks of Schönberner (1983) and Vassiliadis \& Wood (1994) in Fig. 1 (full symbols - post-AGB stars). For a comparison we have also plotted spectroscopic results for PNN taken from Méndez et al. (1992), McCarthy et al. (1997), Napiwotzki (1999), and Rauch et al. (1999) (open symbols in Fig. 1).

The masses of pAGB, as derived by comparing the observed positions to the theoretical tracks in Fig. 1, display a narrow distribution peaking at $0.56 M_{\odot}$. The distribution has, however, a high mass tail which results from cool objects in Fig. 1. This can be considered as consistent with evolutionary expectations. As shown in Tylenda et al. (2001) high mass pAGB are expected to be predominantly observed in F-G spectral classes. On the other hand, the cool pAGB in Fig. 1 are often pulsating variables. It is not clear to what extent this fact can affect the spectroscopic results.

The mean mass for pAGB is $0.589 M_{\odot}$. This is very close to the mean mass for PNN which for the sample displayed in Fig. 1 is $0.600 M_{\odot}$. However, as can be seen from Fig. 1, there is a systematic difference between low $g$ and high $g$ PNN. For instance, for PNN having $\log g \lesssim 6.0$ the mean mass is $0.654 M_{\odot}$ while a value of $0.545 M_{\odot}$ is obtained when PNN with $\log g \gtrless 6.0$ are considered. Thus the masses of pAGB are between the masses of low $g$ and high $g$ PNN.

This result does not agree with expectations from evolutionary considerations. Low mass stars evolve slowly after having left the AGB and should be predominantly observed as pAGB and cool, low $g$ PNN. Their nebulae have enough time to become dispersed in interstellar medium before the stars reach high $T_{\text {eff }}$ and high $g$. Massive stars evolving quickly across the H-R diagram should be seen mainly as hot, high $g$ PNN. Thus the mean observed mass should 


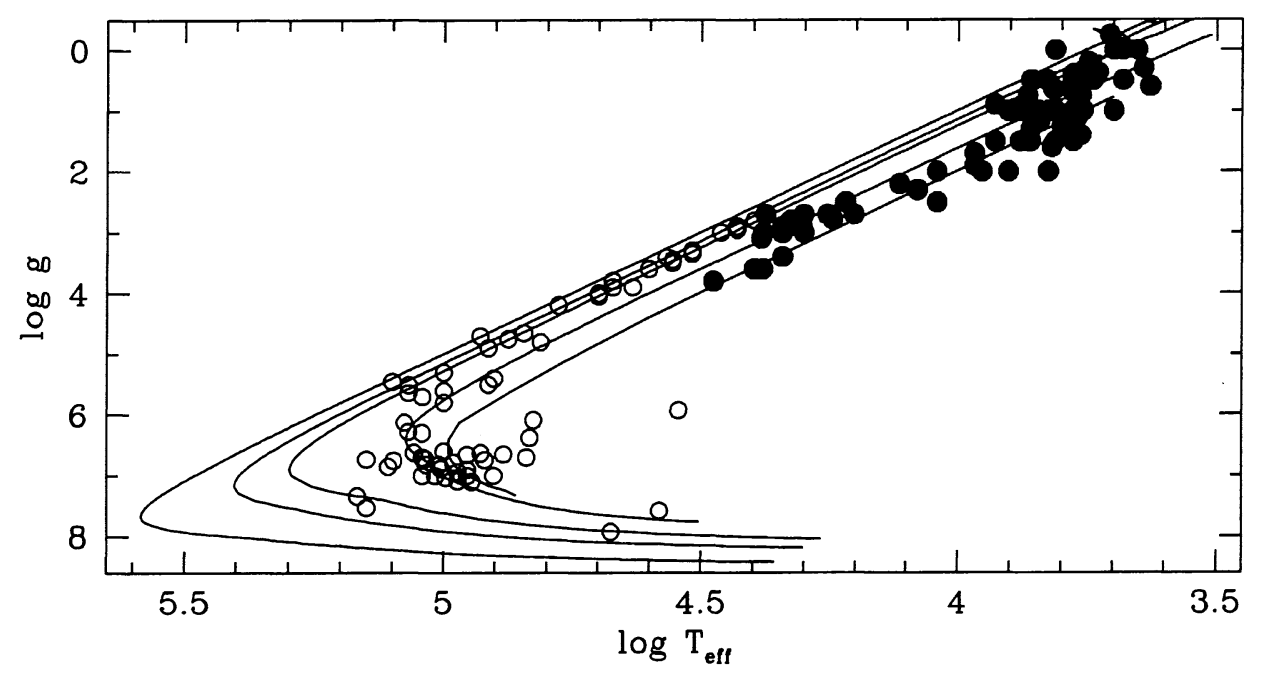

Figure 1. Observed positions of pAGB (full symbols) and PNN (open symbols) in the $\log g-\log T_{\text {eff }}$ diagram. Full curves show theoretical tracks for masses of $0.546,0.569,0.677,0.754$, and $0.900 M_{\odot}$ (from right to left) from Schönberner (1983) and Vassiliadis \& Wood (1994).

be smallest for pAGB, somewhat higher for cool, low $g$ PNN and highest for hot, high $g$ PNN. It is not clear what is the reason for this discrepancy between the theoretical predictions and the observations.

Acknowledgments. The reported research has partly been supported from the Polish State Committee for Scientific Research grant no. 2.P03D.020.17.

\section{References}

McCarthy, J.K., Méndez, R.H., \& Kudritzki, R.-P. 1997, in IAU Symp. 180, Planetary

Nebulae, ed. H.J. Habing \& H.J.G.L.M. Lamers, (Dordrecht: Kluwer), 120

Méndez, R., Kudritzki, R.P., \& Herrero, A. 1992, A\&A, 260, 329

Napiwotzki, R. 1999, A\&A350, 101

Rauch, T., Köppen, J., Napiwotzki, R., \& Werner, K. 1999, A\&A, 347, 169

Schmidt, M. 2001, in Post-AGB Objects as a Phase of Stellar Evolution, ed. R. Szczerba \& S.K. Górny, (Dordrecht: Kluwer), ASSL vol. 265, 271

Schönberner, D. 1983, ApJ, 272, 708

Tylenda, R., Jeske, K., Szczerba, R., \& Siódmiak N. 2001, in Post-AGB Objects as a Phase of Stellar Evolution, ed. R. Szczerba \& S.K. Górny, (Dordrecht: Kluwer), ASSL vol. 265, 139

Vassiliadis, E., \& Wood, P. 1994, ApJS, 92, 125 